\title{
DESIGN AND MEASUREMENTS OF A DAMPING RING KICKER FOR THE ILC
}

\author{
M.J. Barnes", G.D. Wait, TRIUMF, Vancouver, B.C., Canada
}

\begin{abstract}
The International Linear Collider (ILC) requires ultra fast kickers for the damping ring. The modulators must produce pulses of $\pm 5 \mathrm{kV}$ magnitude, with rise and fall times of $6 \mathrm{~ns}$ or less depending on the final configuration [1]. The deflector plates will be configured as a $50 \Omega$ stripline, charged to opposite polarities, providing a potential difference of $10 \mathrm{kV}$. The pulse magnitude must be repeatable to a high accuracy. The need for $3.25 \mathrm{MHz}$ burst mode operation, at $5 \mathrm{~Hz}$, gives an average repetition rate of up to $16.3 \mathrm{kHz}$ [2]. This paper describes a novel design for a pulse generator for the damping ring kickers, in which two stacks of $1 \mathrm{kV}$ FETS will be combined to generate the fast pulses. Each stack of FETs is a $100 \Omega$ driver: the two stacks form a $50 \Omega$ pulse generator as this is preferable to one $50 \Omega$ stack. Measurements and calculations are presented on the present state of the TRIUMF prototype pulse generator.
\end{abstract}

\section{INTRODUCTION}

The 2005 ILC damping ring baseline considered a damping ring with bunch spacing in the range $3 \mathrm{~ns}$ to $14 \mathrm{~ns}$ [1]. However a recent baseline suggests a pulse width of $\leq 4.16 \mathrm{~ns}$, with a burst mode of $3.25 \mathrm{MHz}$, and voltage fluctuations of individual kickers of $\leq 0.33 \%$ [2].

The injection/extraction kickers have to provide $0.6 \mathrm{mrad}$ deflection of $5 \mathrm{GeV}$ bunches. The beam bunches will travel in the opposite direction to the stripline pulse and thus the total deflection $(\theta)$ will be the sum of the magnetic $\left(\theta_{B}\right)$ and electric $\left(\theta_{E}\right)$ components of the deflection. The total deflection is given by:

$\theta=\theta_{B}+\theta_{E}=\frac{0.3 \cdot V \cdot l_{s}}{d \cdot p \cdot c}(1+1 / \beta)$

where $V$ is the potential difference between the stripline plates, $l_{s}$ is the overall length of stripline plates, $d$ is the plate separation, $\beta \cdot c$ is particle velocity, $c$ is the velocity of light in free space $\left(3 \times 10^{8} \mathrm{~m} / \mathrm{s}\right)$, and $p$ is the beam momentum in units of $\mathrm{GeV} / \mathrm{c}$. In this case $\beta=1$ so that the electric and magnetic deflections are equal.

To allow for adequate rise-time of the electrical pulse, sets of striplines mechanically in series are required. To minimize the effective field rise-time, the sets of striplines must be energized at the appropriate time [3]. The permissible pulse rise-time $\left(t_{p}\right)$ is given approximately by:

$t_{p}=t_{b}-2 \cdot l_{s} /(N \cdot c)$

where, $\left(t_{b}\right)$ is the beam gap and $N$ is the number of sections into which the total stripline length is subdivided. If the beam gap is $6 \mathrm{~ns}$ and each set of striplines

\footnotetext{
\# Now employed at CERN, Switzerland.

* Work supported by a contribution from the National Research

Council of Canada.
}

is $30 \mathrm{~cm}$ long $\left(l_{s} / N\right)$, i.e. $1 \mathrm{~ns}$ fill time, $t_{p}=4 \mathrm{~ns}$. To achieve $0.6 \mathrm{mrad}$ deflection, approximately 20 such kickers are needed with the plates pulsed to $\pm 5 \mathrm{kV}$.

\section{DRIVER CONCEPTS}

The TRIUMF kicker group has developed a new circuit concept for a prototype damping ring kicker for ILC. The design of the ILC modulator is based on previous designs at TRIUMF (Table 1) but with significant modifications. All of these previous designs employed 2 stacks of $1 \mathrm{kV}$ FET modules operating in push-pull mode. The MuLan kicker design $[4,5]$ permits operation from DC up to more than $75 \mathrm{kHz}$ continuous at voltages of up to $\pm 12.5 \mathrm{kV}$ into a capacitive load. The RFQ pulser [6] for TITAN has been tested at $600 \mathrm{~V}$ peak-peak at $2.2 \mathrm{MHz}$, and $500 \mathrm{~V}$ peak-peak at $3.0 \mathrm{MHz}$ continuous operation.

Proof of principal tests for the ILC damping ring kickers were performed using the MULAN prototype kicker as a test bed and components on hand, including DE375-102N12A FETs and DEIC420A FET drivers. A $3.1 \Omega$ resistance, previously connected between the driver output and FET gate [4], was reduced to $0.5 \Omega$ to increase transient gate-current. Pulses of $4.6 \mathrm{kV}$, with 6 ns rise and fall times (10\% to $90 \%)$ and $21 \mathrm{~ns}$ width (at the $5 \%$ level), were generated across a $100 \Omega$ load at $1 \mathrm{kHz}$. Subsequently the FET modules, the backplane which the modules plug into, and the mounting stand were redesigned: a module and its backplane connection is termed a level. The goal of the redesign was to reduce pulse width by decreasing inductance and capacitance of each level while maintaining adequate airflow for cooling. Table 1. Measured values for TRIUMF MOSFET kickers

\begin{tabular}{|c|c|c|c|}
\hline $\begin{array}{c}\text { Pulse } \\
\text { voltage }\end{array}$ & $\begin{array}{c}\text { Rise and } \\
\text { fall time }\end{array}$ & $\begin{array}{c}\text { Repetition rate } \\
\text { (continuous) }\end{array}$ & References \\
\hline $6 \mathrm{kV}$ & $30 \mathrm{~ns}$ & $10 \mathrm{~Hz}$ to $20 \mathrm{kHz}$ & See [4] \\
\hline $10 \mathrm{kV}$ & $40 \mathrm{~ns}$ & $10 \mathrm{~Hz}$ to $1 \mathrm{MHz}$ & See [4] \\
\hline $\pm 10 \mathrm{kV}$ & $100 \mu \mathrm{s}$ & $<10 \mathrm{mHz}$ to $10 \mathrm{~Hz}$ & See [4] \\
\hline$-3.5 \mathrm{kV}$ & $63 \mathrm{~ns}$ & DC to $52.2 \mathrm{kHz}$ & See [4] \\
\hline $\pm 12.5 \mathrm{kV}$ & $40 \mathrm{~ns}$ & DC to $77 \mathrm{kHz}$ & {$[4]$} \\
\hline $500 \mathrm{~V}$ & $120 \mathrm{~ns}$ & $3 \mathrm{MHz}$ & {$[6]$} \\
\hline
\end{tabular}

\section{ILC Pulser Concept}

As a result of the relatively low impedance of the damping ring kickers, the proposed design does not require push-pull operation of stacks to achieve fast rise and fall times. The prototype ILC kicker consists of a stack of 15 series, $1 \mathrm{kV}$, modules configured to form a delay line. Each level has a capacitance to ground $(C)$ that is almost independent of its position in the stack, with the exception of the end modules which have an additional $\sim 1 \mathrm{pF}$ to ground. The characteristic impedance $(Z)$ of each level of the stack is given by Eq. 3, where $L$ is the series 
inductance of a level of the stack.

$$
Z=\sqrt{L / C}
$$

The propagation time of a pulse through each level $(\tau)$ is given by

$$
\tau=\sqrt{L \cdot C}
$$

The rise and fall time of the pulse generated is related to the intrinsic switching time of each FET, the number of series FETs (15) and the pulse propagation time through each level of the delay line. The number of series FETs is dependent upon the voltage rating of each FET and the required voltage rating of the stack: FETs with a switching time of $2 \mathrm{~ns}$ to $3 \mathrm{~ns}$ [5] are available up to $1 \mathrm{kV}$. To achieve a fast rising/falling pulse it is necessary to minimize propagation delay and hence inductance. The DEI FETs used have a low inductance, hence the stack inductance is mainly dependent upon the backplane connection between FETs. Eq. 3 and Eq. 4 show that, for a given minimum inductance, the delay of a $100 \Omega$ line is less than that of a $50 \Omega$ line and hence the stack was configured to have an impedance of $\sim 100 \Omega$.

The deflection is independent of the impedance of the striplines and the deflections due to the electric and magnetic fields are equal for $\beta=1$ (Eq. 1). To make use of commercially available components, such as coaxial cable and feed-throughs, it is preferable to select a system impedance of $50 \Omega$ : a driver impedance of $50 \Omega$ can be achieved with 2 parallel $100 \Omega$ stacks.

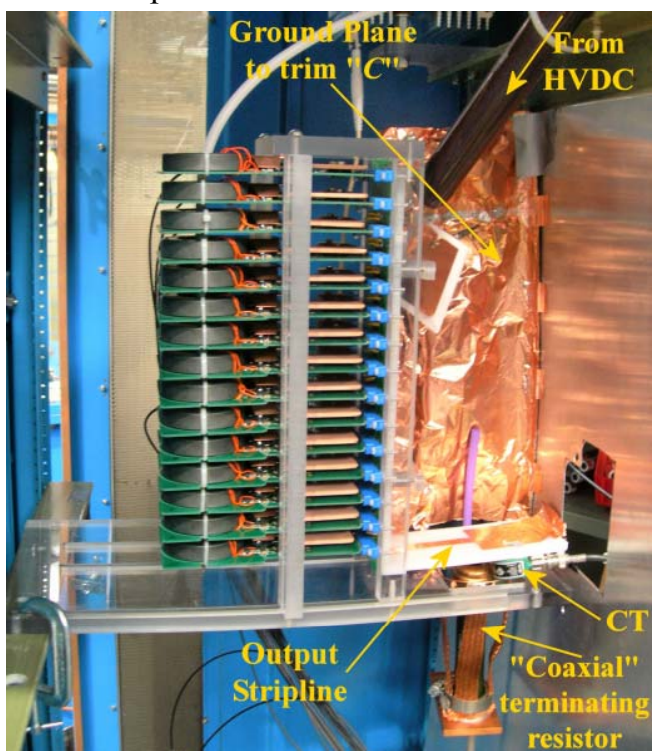

Figure 1: Stack of 15 series FETs configured as a $100 \Omega$ delay line (no heat sinks mounted on cards).

The FET stack (Fig. 1) is charged, from a DC supply, through a $10 \mathrm{k} \Omega$ resistor connected to the drain of the top FET. The source of the bottom FET is connected to a "coaxial" $104 \Omega$ terminating resistor. The stack is charged and subsequently the 15 FETs are turned on, within $0.7 \mathrm{~ns}$ of each other, to discharge the energy stored in the capacitance of the stack into the terminating resistor. The FET stack is turned off $\sim 160$ ns later when there is negligible current flow and the stack is then recharged. The minimum on-time of $160 \mathrm{~ns}$ is a limitation of the present fiber optics, used to trigger the FETs, and will be addressed for $3.25 \mathrm{MHz}$ burst mode operation.

\section{Measurement of Driver Parameters}

Measurements were made on the prototype stack to determine the value of parameters $\tau, L, C$ and $Z$. The value of $\tau$ was determined by measuring the propagation delay through the 15 series levels. The FETs were all held in the on-state and an $8 \mathrm{~V}$ pulse was fed through a $50 \Omega$ coax to the drain of the top FET. Given that the impedance of the stack was close to $100 \Omega$, another $100 \Omega$ resistor was placed in parallel with the stack to provide a reasonable match to the $50 \Omega$ source impedance of the pulse generator. The measured propagation time through 15 levels was $3.1 \mathrm{~ns}$, which corresponds to $0.21 \mathrm{~ns} / \mathrm{level}$.

The inductance of the stack $(15 \cdot L)$ is given by;

$15 \cdot L=\frac{1}{I} \int\left(V_{\text {in }}-V_{\text {out }[\text { in }]}\right) \cdot d t$

where $I$ is the load current, $V_{i n}$ is the input voltage to the stack, and $V_{\text {out }[i n]}$ is the output voltage of the stack normalized to the input voltage. Normalization of the output voltage accounts for the on-state resistance of the FETs. The resultant inductance of the stack is $450 \mathrm{nH} \pm 25 \mathrm{nH}$, which corresponds to $30 \mathrm{nH} \pm 1.7 \mathrm{nH}$ per level. The spacing between modules on the backplane (Fig. 2) is $20 \mathrm{~mm}$ which accounts for $\sim 20 \mathrm{nH}$ and the remaining $10 \mathrm{nH}$ is due to the FET and the power traces on the module.

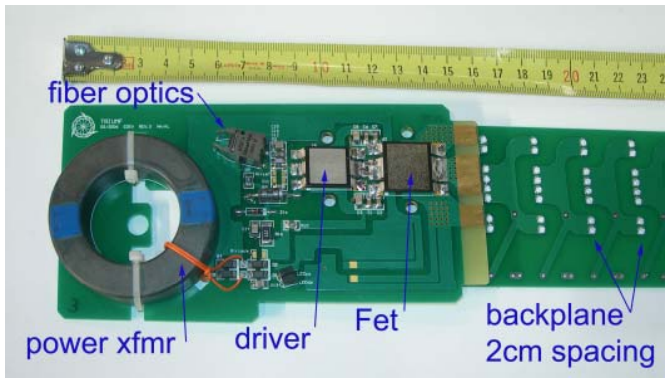

Figure 2: Low inductance FET module and backplane.

The stack capacitance was determined by charging it through a $5.32 \mathrm{k} \Omega$ resistor and measuring the $\mathrm{RC}$ time constant: the FETs were in the on-state and the output of the stack was open-circuit. The average, of $1.75 \mathrm{pF} / \mathrm{level}$, is in good agreement with the theoretical value [5].

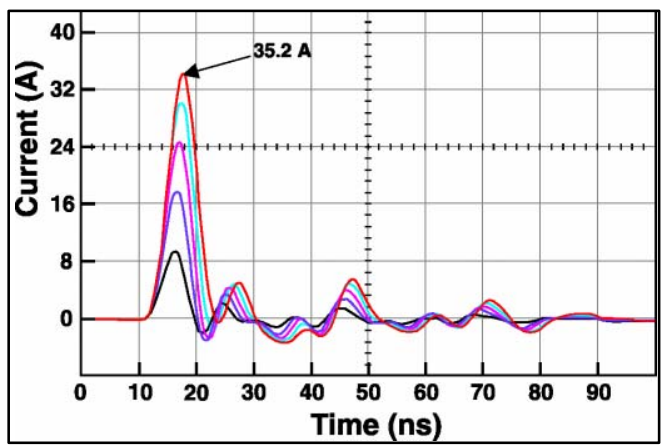

Figure 3: Pulse for $2.5 \mathrm{kV}, 5 \mathrm{kV}, 7.5 \mathrm{kV}, 10 \mathrm{kV}$, and $12.5 \mathrm{kV}$ DC supply; $C=1.75 \mathrm{pF}$ (before increasing capacitance).

$L=30 \mathrm{nH} \& C=1.75 \mathrm{pF}$ corresponds to $Z=130 \Omega$ and T16 Pulsed Power Technology 
$\tau=0.23 \mathrm{~ns} /$ level: this delay is consistent with the direct measurement of $0.21 \mathrm{~ns} /$ level. Fig. 3 shows measured HV pulses: the post-pulse reflections are due to impedance mismatches. Charging the FET stack to $12.5 \mathrm{kV}$ gives 35.2 A peak (measured using a Pearson $2877 \mathrm{CT}$ with a specified useable rise-time of $2 \mathrm{~ns}$ and a $500 \mathrm{MHz}$ Tektronix TDS 7104 oscilloscope) into a $104 \Omega$ load. This corresponds to a peak load voltage of $3.66 \mathrm{kV}$. The amplitude is lower than $12.5 \mathrm{kV} / 2$ because the stack impedance is high, the FETs have an on-state resistance of $\sim 1 \Omega$ each and the parasitic capacitances to ground of each level in the delay line are not all pre-charged to $12.5 \mathrm{kV}$ due to (resistive) voltage grading down the stack.

To decrease $Z, C$ was increased: this was achieved by installing an additional ground plane beside the stack (Fig. 1). By trial and error the magnitude of the post pulse reflections were reduced, which is an indication that the characteristic impedance is matched to the terminator. Remeasuring gave an average $C$ of $3.3 \mathrm{pF} /$ level. Fig. 4 shows the measured HV pulses for $C=3.3 \mathrm{pF}$ with $15 \mathrm{pF}$ added to the drain of the top FET: the pulse magnitude, rise-time and width are increased in comparison with $Z=130 \Omega$ (Fig. 3). The output current magnitude is $46.2 \mathrm{~A}$ $(4.8 \mathrm{kV}$ into $104 \Omega$ ) for $12.5 \mathrm{kV} \mathrm{DC}$. The measured $10 \%$ to $90 \%$ rise-time is $4.3 \mathrm{~ns}$. Assuming that neither the CT nor oscilloscope causes overshoot of the pulse, the actual rise-time of the current can be estimated to be $3.7 \mathrm{~ns}$ [3]. A higher bandwidth measurement system is required to more accurately determine the rise-time of load current.

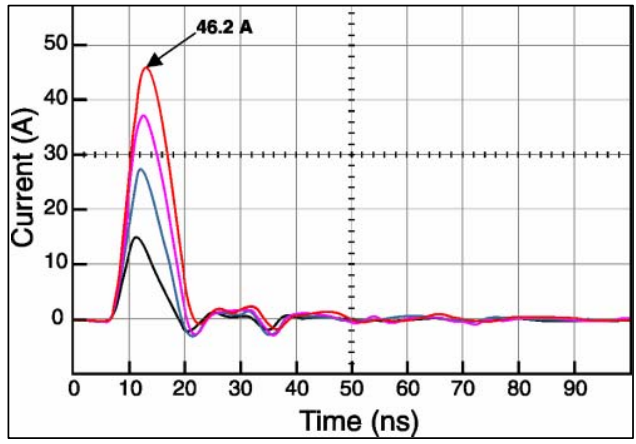

Figure 4: Pulse for $3 \mathrm{kV}, 6 \mathrm{kV}, 9 \mathrm{kV} \& 12.5 \mathrm{kV}$ DC, for $\mathrm{C}=3.3 \mathrm{pF} \&$ with $15 \mathrm{pF}$ added to the drain of the top FET.

In Figs. 3 and 4 the amplitude of the pulse does not increase linearly with the applied DC voltage, however the area of the pulse is proportional to the charge stored on the stack. PSpice also demonstrates the same effect, which is attributable to Miller capacitance of the FETs: increasing the gate drive, in the PSpice simulation, reduces the non-linearity. In Fig. 3 the full pulse width varies from $7.7 \mathrm{~ns}$ for $2.5 \mathrm{kV} \mathrm{DC}$ to $10.6 \mathrm{~ns}$ for $12.5 \mathrm{kV}$ DC, whereas in Fig. 4 the full pulse width varies from $11.5 \mathrm{~ns}$ for $3 \mathrm{kV}$ DC to $13.7 \mathrm{~ns}$ for $12.5 \mathrm{kV} \mathrm{DC}$.

The FETS in the stack were deliberately positioned in the order of the measured delay of each FET, with the top FET turning-on $0.7 \mathrm{~ns}$ before the bottom FET. PSpice predictions show that this results in an asymmetrical pulse with a faster rise-time and slower fall-time: if the FETs are all triggered coherently then the predicted pulse is symmetrical. Triggering the FETs, in the $100 \Omega$ stack, in sequence, starting at the top of the stack, such that there is $0.3 \mathrm{~ns}$ between FETs turning-on, compensates for the $\sqrt{L \cdot C}(0.3 \mathrm{~ns})$ delay between levels: hence the rise-time is faster (5.2 ns predicted, $10 \%$ to $90 \%$ ) but the fall-time is longer. If the order of the timing is reversed $(-0.3 \mathrm{~ns}$ in Fig. 5) then a shorter fall-time can be achieved, with a longer rise-time (6.7 ns predicted, $10 \%$ to $90 \%$ ).

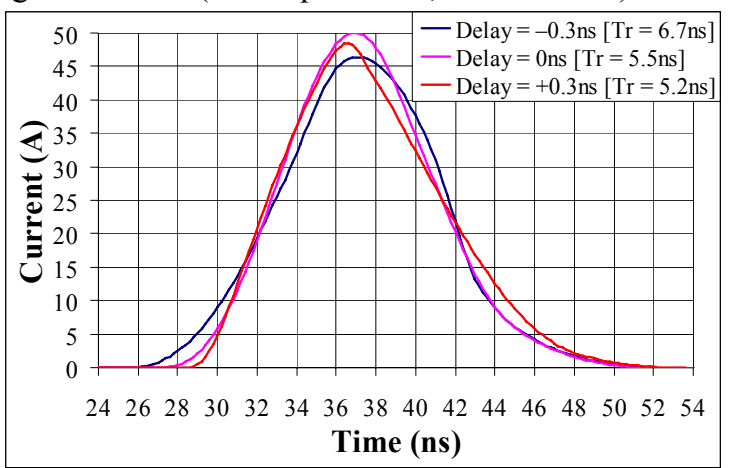

Figure 5: Predicted load current with $-0.3 \mathrm{~ns}, 0 \mathrm{~ns} \&$ $+0.3 \mathrm{~ns}$ delay between adjacent FETs for $12.5 \mathrm{kV}$ DC.

\section{Conclusion}

Pulses of $4.8 \mathrm{kV}$, with $4.3 \mathrm{~ns}$ rise and $5.5 \mathrm{~ns}$ fall, $10 \%$ to $90 \%$, and a width of $13.7 \mathrm{~ns}$ at the $5 \%$ level have been generated at $60 \mathrm{kHz}$ continuous: the post pulse ripple is $< \pm 5 \%$. This is very close to meeting the specifications for the widest (14 ns), 2005 baseline, ILC pulses. The pulse rise-time and width can be further reduced by decreasing the stack inductance, by reducing the FET spacing, and by increasing the gate drive. Fast, higher voltage, FETs would also reduce the delay of the stack and hence the rise-time and width of the pulse. To allow adequate time to recharge the stack capacitance, improved fibre optics are required for the $3.25 \mathrm{MHz}$ burst mode so that the FETs can be turned off within $50 \mathrm{~ns}$ or less.

\section{REFERENCES}

[1] G.F. Dugan, Private Communication, TRIUMF Meeting Nov. 1, 2005.

[2] M.A. Palmer et al., "Tests of a High Voltage Pulser for ILC Damping Ring Kickers", To be Publ. Proc. EPAC'06, 26-30 June 2006.

[3] M.J. Barnes et al., "Design of the Modulator for the CTF3 Tail Clipper Kicker", This conf.

[4] R.B. Armenta, M.J. Barnes \& G.D. Wait, "Precision $25 \mathrm{kV}$ Pulse and RF Suppression in a $75 \mathrm{kHz}$ Kicker", 15th IEEE Int. Pulsed Power Conf., June 13-17 2005, Monterey, USA, pp676-679.

[5] M.J. Barnes \& G.D. Wait, "A 25 kV, 75 kHz, Kicker for Measurement of Muon Lifetime", IEEE Trans. Plasma Science, Vol. 32, No. 5, Oct. 2004, pp19321944.

[6] M.J. Barnes et al., "A High Frequency MOSFET Driver for the TITAN Facility at TRIUMF", Proc. 15th IEEE Int. Pulsed Power Conf., June 13-17 2005, Monterey, USA, pp178-181. 